\section{Familial amyloidosis cutis dyschromica in three siblings: report from Indonesia}

\author{
Melyawati Hermawan, Rahadi \\ Rihatmadja, Sondang Pandjaitan Sirait \\ Department of Dermatovenereology, \\ Faculty of Medicine, Universitas \\ Indonesia, Dr. Cipto Mangunkusumo \\ General Hospital, Jakarta, Indonesia
}

\begin{abstract}
Amyloidosis cutis dyschromica (ACD) is an extremely rare type of primary cutaneous amyloidosis. To date there are fewer than 40 published cases worldwide; some were reported affecting several family members. Its resemblance to other common pigmentation disorders makes it rarely recognized at first sight. Our patient, the 12-yearold firstborn son of non-consanguineous parents presented with generalized mottled pigmentation starting from lower extremities. His siblings suffered from similar condition. The clue for diagnosis is the amyloid deposition in the papillary dermis. The etiology of ACD is still unknown, but genetic factors and ultraviolet radiation are implicated. It is proposed that disturbance of keratinocyte repair following ultraviolet radiation results in amyloid deposition. The treatment remains a challenge. Oral acitretin treatment, thought to repair keratinization defect, gave a slight improvement in our case. Our is the first case of ACD reported in Indonesia.
\end{abstract}

\section{Introduction}

Cutaneous amyloidosis were classified into primary cutaneous amyloidosis (PCA), secondary cutaneous amyloidosis, and systemic cutaneous amyloidosis. PCA is a rare, chronic progressive skin disease, defined as deposition of amyloid in previously apparent normal skin without systemic involvement. ${ }^{1,2}$ The clinical features of PCA are varied. Well-known clinical types of PCA were macular, papular, and nodular. ${ }^{2}$ Amyloidosis cutis dyschromica (ACD) is a rare type of PCA. ${ }^{1}$ Its prevalence were rarely reported. Until now, there are fewer than 40 published cases worldwide. Etiological factors associated with PCA is still unknown, but its striking familial occurence suggests the role of genetic. ${ }^{1,3}$ The treatment of ACD is not well documented.

We report a case with peculiar mottled pigmentation originally referred for vitiligo, but later proved as ACD by histopathological examination. The disorder was thought to be familial as his siblings were affected with the similar condition. Treatment with oral acitretin gave satisfying result.

\section{Case Report}

A 12-year-old boy presented with asymptomatic, generalized mottled hypo- and hyper-pigmented lesions of 6 -year duration. The hypopigmented macules were first noticed on his lower extremities and had been slowly progressing to involve almost the entire body (Figure 1A). He did not have any history of systemic or cutaneous disease before the onset of the lesions. History of trauma, rubbing of the skin with any material, and extensive sun exposure was denied. Developmental milestones were normal. His 11-year-old sister and 6-year-old brother experienced similar yet milder symptoms. They were born to non-consanguineous parent.

Physical examination revealed an extensive, discrete, pigmented macules distributed nearly all over the body in symmetrical pattern. The pigmentation was intermixed with small hypopigmented macules. There was no apparent papular, erythema, atrophy, or telangiectasia. The hair, teeth, nails, and mucosa had normal appearance. He was referred to our hospital for skin biopsy with the initial differential diagnoses of vitiligo and pityriasis alba. Histopathological examination (Figure 2) showed uneven distribution of melanin in the epidermis and deposits of pale pink amorphous material in the papillary dermis. These deposits showed weak positive stainng with congo red which indicated an amyloid substance. Routine blood tests, biochemical profile, and urinalysis were within normal limits. Based on clinical presentation and the histopathology examination, we made the diagnosis of primary cutaneous amyloidosis, presented as amyloid cutis dyschromica. The patient was treated with oral acitretin $25 \mathrm{mg}$ per day. By the third month, some improvement was observed as the pigmented macules were slightly lightened (Figure 1B). The patient could tolerate the treatment, as there was no significant increase of transaminases and the lipid profile.

\section{Discussion}

The diagnosis of ACD in our patient was not readily recognized as it mimicked, to some degree, other relatively common disorders with pigmentation feature. That, combined with its low prevalence, had eluded previous attempts at the correct diagnosis and treatment. The peculiar asymptomatic mottled pigmentation is much likely seen in poikiloderma, but as our case showed, without the corresponding signs such as telangiectasia or atrophy. Furthermore, our
Correspondence: Melyawati Hermawan, Department of Dermatovenereology, Faculty of Medicine, University of Indonesia - Dr. Cipto Mangunkusumo General Hospital, Jalan Diponegoro no 71, Jakarta Pusat, Indonesia. Tel.: +62.21.786.7222

E-mail: dr_melyawati@yahoo.com

Key words: amyloidosis cutis dyschromica, familial, histopathology, acitretin.

Acknowledgements: the authors would thank Dr. Sri Adi Sularsito, of our institution's Division of Dermatopathology, for his suggestions, and Dr. Triana Agustin and Dr. Githa Rahmayunita of the Division of Pediatric Dermatology for their help in the therapy with acitretin.

Contributions: MH was responsible for performing the clinical examination and skin biopsy, obtaining the family history background, including that of the siblings, and commencing and monitoring the acitretin therapy; RR SPS read the pathology slides and review the clinicopathologic correlation of the case

Conflict of interests: the authors declare no potential conflict of interests.

Conference presentation: this paper had been presented in an oral session on 13th Annual Scientific Meeting of Indonesian Society of Dermatology and Venereology; 23-26 0ctober 2013, Jakarta, Indonesia.

Received for publication: 27 February 2014.

Revision received: 1 August 2014.

Accepted for publication: 26 October 2014.

This work is licensed under a Creative Commons Attribution NonCommercial 3.0 License (CC BYNC 3.0).

(C) Copyright M. Hermawan et al., 2014

Licensee PAGEPress, Italy

Dermatology Reports 2014; 6:5375

doi:10.4081/dr.2014.5375

patient was otherwise healthy, showing no signs that might indicate systemic disorders or photosensitivity, e.g. dermatomyositis, lupus erythematosus, or xeroderma pigmentosum. As defined by Morishima, ACD characterized by diffuse speckled hyperpigmentation with hypopigmented spots without papulation, atrophy, and telagiectasia, mild or no itching, onset before puberty, and focal amyloid deposition in the papillary dermis. ${ }^{4}$ Our patient met all of the criteria. However, another disease with clinically similar interspersed pigmentation - and similarly rare dyschromatosis universalis hereditaria (DUH), merit our consideration. DUH begins in infancy or childhood, and unlike acropigmentation of Dohi, might encompass the whole skin surface with exception of face. ${ }^{5}$ For definite diagnosis, histopathologic examination should be shought. 


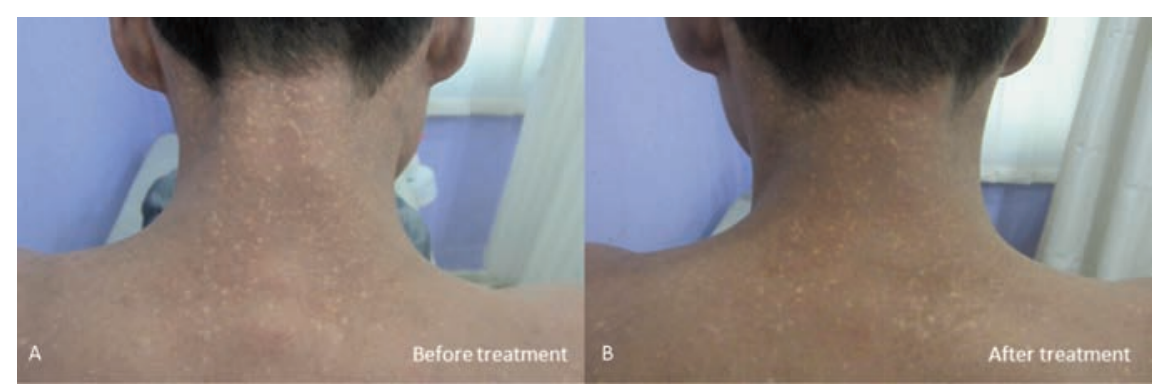

Figure 1. Nuchae before treatment (A) and after treatment (B) with some improvement.

A

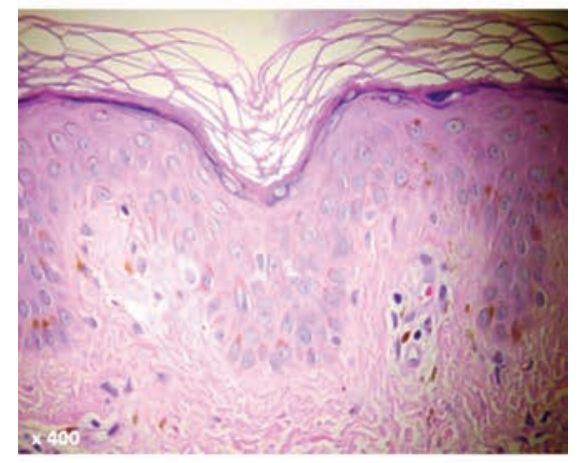

B
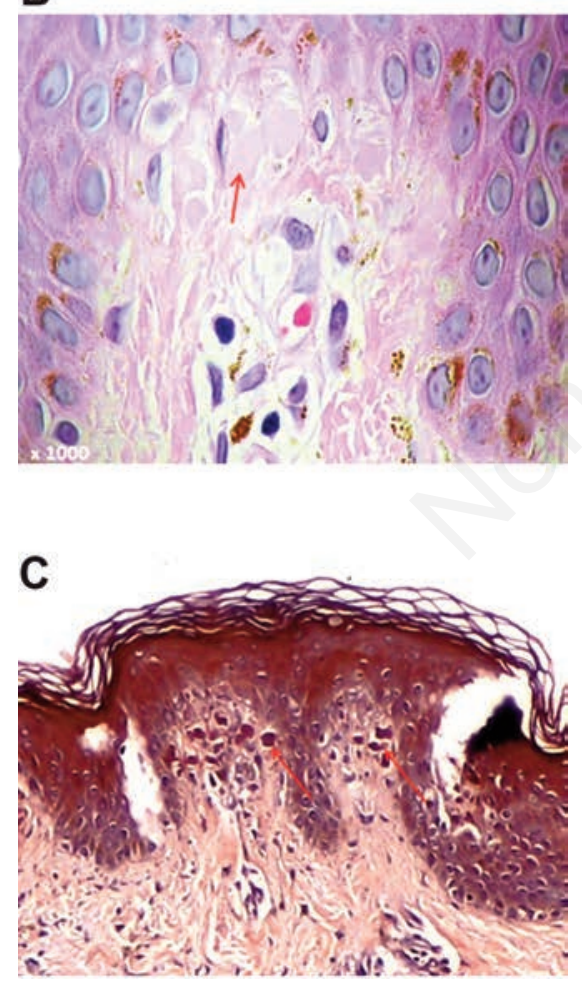

Figure 2. A,B) Histopathological examination showed uneven distribution of melanin in the epidermis and deposits of pale pink amorphous material (red arrow) in the papillary dermis. (H\&E staining). C) Deposits showing positive reaction with Congo Red (400x).
The most common epidermal findings of PCA were hyperkeratosis, irregular acanthosis with thinning of rete ridges, and expansion of dermal papillae by amyloid deposition. The finding of amyloid bodies in the papillary dermis was crucial in establishing the diagnosis of ACD in our patient and disproved DUH, in which such deposition was absent. ${ }^{5,6}$ Its visualization under polarized light, showing apple-green birefrigence, confirmed the presence of amyloid. Special histochemical stains were helpful for confirming the existence of amyloid. ${ }^{7}$ In our case, amyloid bodies were weakly positive with congo red staining. We tried to obtain histopathological examination of the siblings, but the parents denied the request to perform biopsy on the grounds that their clinical appearances were quite similar that the histopathological findings would likely be the same. The etiology of ACD is still not known, but it might be multifactorial. Multiple factors such as race, genetic, and enviromental may play collective roles, making variable degrees of cutaneous amyloidosis. ${ }^{8}$ Although most cases of ACD were sporadic, many have also reported positive family history of PCA, suggesting that the important role of genetic factors in its pathogenesis. ${ }^{1,3}$ Our patient's siblings experienced similar yet milder symptoms that the disorder was thought to be familial. However, consanguinity in family background was denied. In one series, familial relationship was found in 5 of 10 patients, and consanguinity was denied in all. ${ }^{6}$ So far, the genetic locus responsible for the disorder is still under investigation. ${ }^{1}$ Amyloidosis cutis dyschromica is assumed to be a congenital disorder and exposure to sunlight is thought to be the major causal factor. There is a possibility of DNA repair defects caused by sensitivity to UV radiation. ${ }^{9}$ The lesions in our patient were more pronounced on exposed parts of body, that we thought sun exposure might be an important cause. Many therapeutic modalities have been proposed with variable success. Acitretin was given and seems to be effective because it may act by minimizing keratinization defect that causes keratin degeneration to amyloid as proposed by some to be the pathogenesis of this disorder. ${ }^{10}$ Qiao et al. in his series has convincingly shown $100 \%$ positivity to immunohistochemical staining for keratin, CK34 E12 and CK5/6, that the amyloid is thought to be of epidermal origin. ${ }^{6}$ This finding seems to agree with the work of Moriwaki et al..$^{9}$ that points at a disturbance of keratinocyte repairs after irradiation with ultraviolet. At the third month, some improvement was obtained in our patient as the mottled pigmentation became lightened. His serum transaminase and lipid profile were only slightly increased, that we think the drug was safe to be continued. The patient and his mother were satisfied with the result.

\section{Conclusions}

To our knowledge, this is the first case of ACD reported in Indonesia. However, it is interesting to note that some authors consider certain populations of Asia, e.g. Chinese, Japanese and Thai, particularly susceptible. ${ }^{6}$ It hasn't been demonstrated yet wether the same is true within Indonesian population. Therefore, it is possible that several cases of this elusive disorders exist, but are often misdiagnosed. On the other hand, the treatment of ACD still remains a challenge.

\section{References}

1. Yang W, Lin Y, Lin W. Amyloidosis cutis dyschromica in two female siblings: case report. BMC Dermatol 2011;11:1-4.

2. Schreml S, Szeimies RM, Vogt T, et al. Cutaneous amyloidoses and systemic amyloidoses with cutaneous involvement. Eur J Dermatol 2010;20:152-60.

3. Wong LP Tang YM, Lai WY. Clinical pattern of primary cutaneous amyloidosis in Hong Kong Chinese. Hong Kong J Dermatol Venereol 2009;17:129-35.

4. Morishima T. A clinical variety of localized cutaneous amyloidosis characterized by dyschromia (amyloidosis cutis dyschromica). Jpn J Dermatol B 1970;80:43-52.

5. Kenani N, Ghariani N, Denguezli M, et al. Dyschromatosis universalis hereditaria: two cases. Dermatol Online J 2008;14:16.

6. Qiao J, Fang H, Yao H. Amyloidosis cutis dyschromica. Orphanet J Rare Dis 2012;7:1-6.

7. Vijaya B, Dalal BS, Manjunath GV. Primary cutaneous amyloidosis: a clinico-pathological study with emphasis on polarized microscopy. Indian J Pathol Microbiol 2012;55:170-4.

8. Eswaramoorthy V, Kaur I, Das A, Kumar B. Macular amyloidosis: etiological factors. J Dermatol 1999;26:305-10.

9. Moriwaki S, Nishigori C, Horiguchi Y, et al. Amyloidosis cutis dyschromica: DNA repair reduction in the cellular response to UV light. Arch Dermatol 1992;128:966-70.

10. Ozcan A, Senol M, Aydin NE, Karaca S. Amyloidosis cutis dyschromica: a case treated with acitretin. J Dermatol 2005;32:474-7. 